\title{
Coherent control of a classical nanomechanical
}

\section{two-level system}

\author{
T. Faust, J. Rieger, M. J. Seitner', J. P. Kotthaus and E. M. Weig $\star^{\dagger}$
}

The Bloch sphere is a generic picture describing the coherent dynamics of coupled classical or quantum-mechanical twolevel systems under the control of electromagnetic fields ${ }^{1,2}$. It is commonly applied to systems such as spin ensembles ${ }^{3}$, atoms $^{4}$, quantum dots $^{5}$ and superconducting circuits ${ }^{6}$. The underlying Bloch equations ${ }^{7}$ describe the state evolution of the two-level system and allow the characterization of both energy and phase relaxation processes ${ }^{3,8,9}$. Here we realize a classical nanomechanical two-level system ${ }^{2}$ driven by radiofrequency signals. It is based on the two orthogonal fundamental flexural modes of a high-quality-factor nanostring resonator that are strongly coupled by dielectric gradient fields ${ }^{10}$. Full Bloch sphere control is demonstrated by means of Rabi ${ }^{11}$, Ramsey ${ }^{12}$ and Hahn echo ${ }^{13}$ experiments. Furthermore, we determine the energy relaxation time $T_{1}$ and phase relaxation times $T_{2}$ and $T_{2}^{*}$, and find them all to be equal. Thus decoherence is dominated by energy relaxation, implying that not only $T_{1}$ but also $T_{2}$ can be increased by engineering larger mechanical quality factors.

Whereas the dynamics of semiclassical two-level systems under the influence of a pulsed external electromagnetic field was observed decades ago in many-spin NMR experiments, a completely classical analogue remained elusive for a long time. Such a classical two-mode system can for example be created using two optical cavity modes $^{14}$ or mechanical resonators. Only recently, several approaches were employed to achieve purely mechanical resonant coupling either between separate resonators ${ }^{15-17}$ or different modes of the same resonator ${ }^{10,18}$ in the classical regime. So far, the pulsed coherent control of the system was prevented by weak coupling, low quality factors or the lack of a sufficiently strong and fast tuning mechanism.

We present the successful implementation of a purely mechanical, classical two-level system, consisting of the two coupled fundamental flexural modes of a nanomechanical resonator with coherent time-domain control (see also the experiments independently performed using parametric coupling ${ }^{19}$ instead of the linear coupling employed here). To this end, we use a $250 \mathrm{~nm}$ wide and $100 \mathrm{~nm}$ thick, strongly stressed ${ }^{20}$ silicon nitride string resonator with a length of $50 \mu \mathrm{m}$ dielectrically coupled to a pair of electrodes used for detection ${ }^{21}$ as well as actuation and tuning ${ }^{22}$. The two fundamental flexural modes of the mechanical resonator oscillating in the out-of-plane and in-plane direction (Fig. 1a) are linearly coupled by cross-derivatives of the strong inhomogeneous electric field generated between the electrodes (see the Supplementary Information of ref. 10 for a theoretical analysis). A constant d.c. voltage of $-15 \mathrm{~V}$ is employed to dielectrically tune the system close to the resulting avoided crossing, while the signals generated by an arbitrary waveform generator (AWG) enable time-resolved control

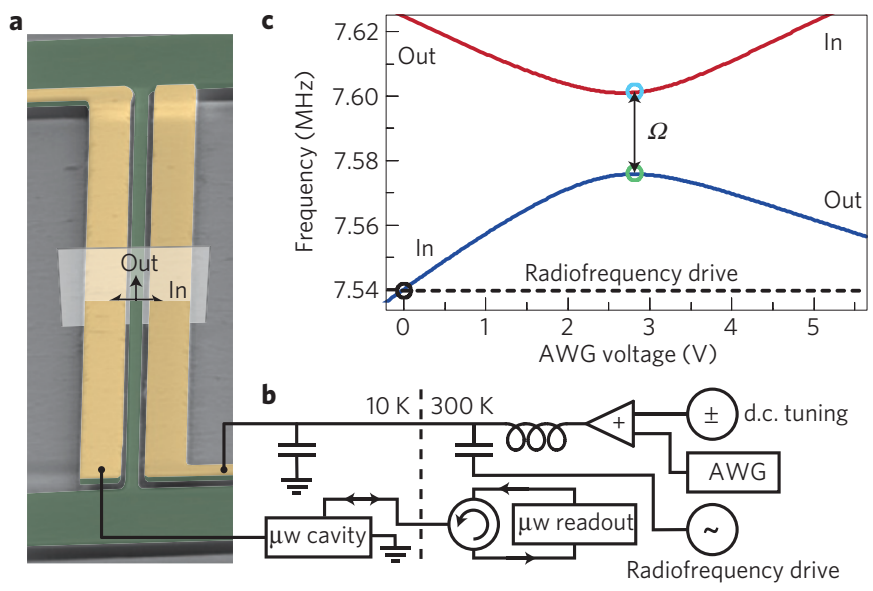

Figure 1 | Nanoelectromechanical system. a, Scanning electron micrograph showing oblique view of the $50 \mu \mathrm{m}$ long silicon nitride string (green) and the adjacent, $1 \mu \mathrm{m}$ wide gold electrodes (yellow), processed on top of the SiN. b, Electrical set-up. The output of the AWG and a d.c. tuning voltage are added and combined with the radiofrequency drive at a bias-tee. The second capacitor acts as a bypass providing a microwave ( $\mu \mathrm{w})$ ground path for the microwave detection ${ }^{21}$. $\mathbf{c}$, Resonance frequencies of the out-of-plane (Out) and in-plane (In) mode of the resonator are controlled by the AWG voltage at a constant d.c. tuning voltage of $-15 \mathrm{~V}$. The black circle marks the initialization state at $0 \mathrm{~V}$ and the frequency of the radiofrequency drive, and the green and blue circles correspond to the lower and upper state of the classical two-level system, respectively, separated by $\Omega$.

in the vicinity of the avoided crossing (Fig. 1b,c). Both voltages are added, combined with the radiofrequency actuation at a bias-tee, and applied to one electrode. The other electrode is connected to a $3.6 \mathrm{GHz}$ microstrip cavity, enabling heterodyne detection of the string deflection ${ }^{21}$ after addition of a microwave bypass capacitor at the first electrode ${ }^{22}$. These components, as well as the mechanical resonator, are placed in a vacuum of $\leq 10^{-4} \mathrm{mbar}$ and the system is cooled to $10.00 \pm 0.02 \mathrm{~K}$ to improve the temperature stability as well as the cavity quality factor. The microwave cavity is interfaced to the readout with a single coaxial cable and a circulator.

When the system is driven by an external white-noise source and the AWG output voltage is swept, the avoided crossing of the two modes shown in Fig. 1c can be mapped out, exhibiting a frequency splitting $\Omega=24,249 \pm 4 \mathrm{~Hz}$. With a quality factor $Q=f / \Delta f \approx 2 \times 10^{5}$ and a linewidth of $\Delta f \approx 40 \mathrm{~Hz}$ at the resonance frequency $f$, the system is clearly in the strong coupling regime of $\Delta f \ll \Omega$. For all measurements discussed in the following, 


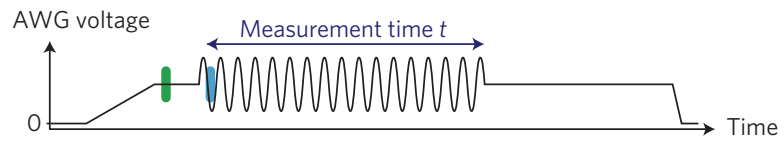

b

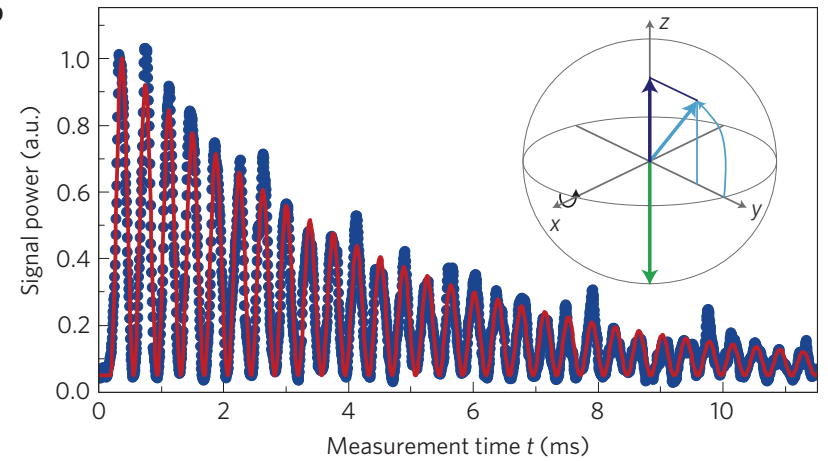

Figure 2 | Rabi oscillations. a, Pulse scheme. The system is adiabatically tuned from the initialization to the lower state, then a constant drive with frequency $\Omega$ is turned on. $\mathbf{b}$, The $z$ projections of the decaying Rabi oscillations (data coloured dark blue; fit indicated in red) can be directly measured with a spectrum analyser. The Bloch sphere in the inset shows the state of the Bloch vector at selected times, which are marked in the same colour in a.

a radiofrequency drive of $-59 \mathrm{dBm}$ at $7.539 \mathrm{MHz}$, resonantly actuating the string at an AWG voltage of $0 \mathrm{~V}$, is applied, which initializes the system in its in-plane mode (Fig. 1c, black circle). A $1 \mathrm{~ms}$ long, adiabatic voltage ramp up to $2.82 \mathrm{~V}$ brings the state to the point of minimal frequency splitting $\Omega$ between the coupled modes. Here, the system dynamics is described by two normal modes, hybrid states formed by the in-phase and out-of-phase combinations of the fundamental flexural modes. The adiabatic ramp thus transforms all the energy of the in-plane mode into the lower hybrid state, such that the classical two-level system ${ }^{2}$, consisting of the two hybrid modes, is prepared in its lower state. As the drive frequency remains constant (Fig. 1c, dashed line), the string is no longer actuated and its energy is slowly decaying.

Now, the AWG is used to apply a continuous pump tone with frequency $\Omega$ to the drive electrodes which starts Rabi oscillations ${ }^{3,11}$ between the lower and upper state, as shown in Fig. 2. They can be measured directly by monitoring the time evolution of the output power spectrum at the frequency of one of the hybrid modes, here shown for the upper state at $7.6028 \mathrm{MHz}$, and measured with a bandwidth of $10 \mathrm{kHz}$. All time-resolved measurements are averaged over 20 (Rabi oscillations and T1 measurement) or 10 pulse sequences (Ramsey fringes and Hahn echo). For a drive amplitude of $100 \mathrm{mV}$ (half peak-to-peak) we find a Rabi frequency of $8.3 \mathrm{kHz}$ (Supplementary Section SIIA). These strong Rabi oscillations demonstrate that the transition between the two hybrid modes forms a classical two-level system, in contrast to the modes themselves, which can be modelled as highly populated harmonic oscillators.

In principle, the decay of these oscillations is governed by both energy relaxation, characterized by a rate $1 / T_{1}$, and phase decoherence, characterized by $1 / T_{2}$ or $1 / T_{2}^{*}$, where $T_{2}^{*} \leq T_{2}$ includes reversible processes caused by slow fluctuations or spatial inhomogeneity of the coupling. For clarity, we use these well-known phenomenological constants in the same way as, for example, in spin systems ${ }^{3}$, as discussed in detail in Supplementary Section SI.

The exponential decay of a state's energy defines $T_{1}$. The corresponding measurement is shown in Fig. 3 for both the lower and upper state: the system is once again prepared in the lower hybrid state. To reach the upper state, a subsequent $\pi$-pulse is applied, thus performing one half of a Rabi cycle, which transfers

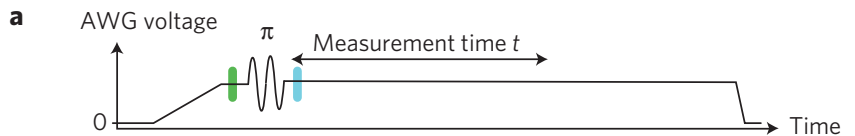

b

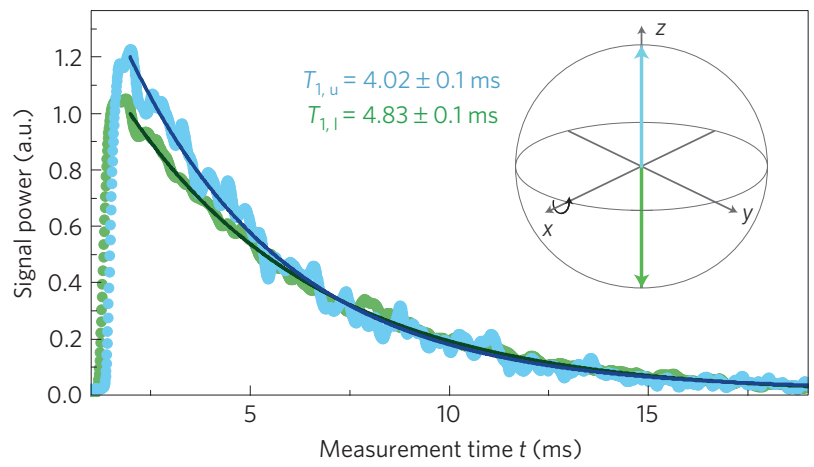

Figure 3 | Energy relaxation. a, Pulse scheme. The system is adiabatically tuned from the initialization to the lower state. An additional $\pi$-pulse is used to rotate it to the upper state. $\mathbf{b}$, Measured exponential decay of the lower (data shown in green; fit in dark green) and upper (data indicated in blue; fit in dark blue) state. The Bloch sphere in the inset shows the state of the Bloch vector at selected times, which are marked in the same colour in a.

the system to the upper state (Supplementary Section SII for details on the frequency and amplitude calibration of the applied pulses). The exponential decay is then measured directly with a spectrum analyser at a bandwidth of $3 \mathrm{kHz}$, exhibiting different relaxation times $T_{1,1}=4.83 \pm 0.1 \mathrm{~ms}$ and $T_{1, \mathrm{u}}=4.02 \pm 0.1 \mathrm{~ms}$ for the lower and upper mode, respectively. They correspond to the spectrally measured quality factors. Previously, it has been shown that, at maximum coupling, the two hybrid modes should have the same quality factor and thus $T_{1}$ time $e^{10}$. However, both modes are affected by dielectric damping ${ }^{22}$ (Supplementary Section SIB), leading to the observed difference.

To measure the $T_{2}^{*}$ time, a $\pi / 2$-pulse is used after the preparation in the lower state to bring the system into a superposition state between the lower and upper hybrid modes. The frequency of the pulse is detuned to $\Omega+500 \mathrm{~Hz}$, leading to a slow precession of the state vector around the $z$ axis of the Bloch sphere ${ }^{3,12}$. As a result, a second $\pi / 2$-pulse after time $\tau$ does not always bring the system into the upper state, but a slow oscillation, the so-called Ramsey fringes, is observed when the delay $\tau$ between the two pulses is varied and the $z$-projection of the state vector is measured after the second pulse, as shown in Fig. 4. The decay constant of this oscillation is $T_{2}^{*}$, whereas the decay of the mean value can be interpreted as an effective $T_{1}$ of both modes. The fit in Fig. $4 \mathrm{~b}$ results in $T_{2}^{*}=4.44 \pm 0.1 \mathrm{~ms}$ and $T_{1}=$ $4.31 \pm 0.1 \mathrm{~ms}$. The energy relaxation time of the superposition state $T_{1}$ is identical to the reciprocal rate average of the two hybrid modes

$$
\overline{T_{1}}=2\left(\frac{1}{T_{1,1}}+\frac{1}{T_{1, \mathrm{u}}}\right)^{-1}=4.39 \mathrm{~ms}
$$

as the mechanical energy oscillates between the two modes with frequency $\Omega$ (Supplementary Movie).

By including an additional $\pi$-pulse at $\tau / 2$ into the Ramsey pulse scheme and replacing the final $\pi / 2$-pulse by a $3 \pi / 2$-pulse to once again rotate to the upper state (Fig. 5), the $T_{2}$ time can be measured in a Hahn echo experiment ${ }^{3,13}$. The $180^{\circ}$ rotation flips the state vector in the $x y$-plane of the Bloch sphere, thus reversing the effects of a fluctuating or inhomogeneous coupling strength $\Omega$ in the second delay interval of $\tau / 2$ and thereby cancelling their contribution. The frequency of the pulses is once again exactly $\Omega$, as all three pulses need to be applied exactly around the same 


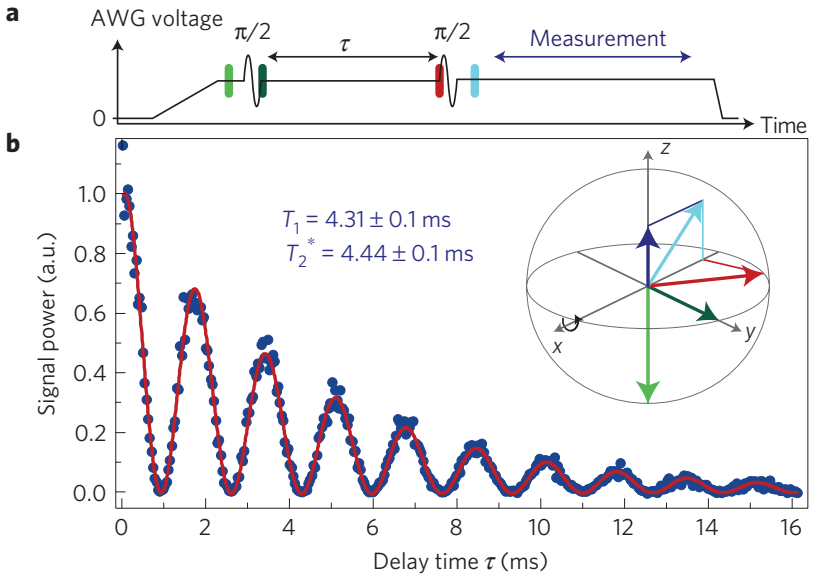

Figure 4 | Ramsey fringes. a, Pulse scheme. The system is adiabatically tuned from the initialization to the lower state. $A \pi / 2$-pulse creates a superposition state, and after a delay $\tau$ a second $\pi / 2$-pulse is applied. b, $\mathrm{A} 500 \mathrm{~Hz}$ detuning between the drive and precession frequency leads to a slow rotation of the superposition state in the equator plane of the Bloch sphere, giving rise to a beating pattern in the measured $z$ component after the second pulse (data shown in dark blue; fit in red). The Bloch sphere in the inset shows the state of the Bloch vector at selected times, which are marked in the same colour in $\mathbf{a}$.

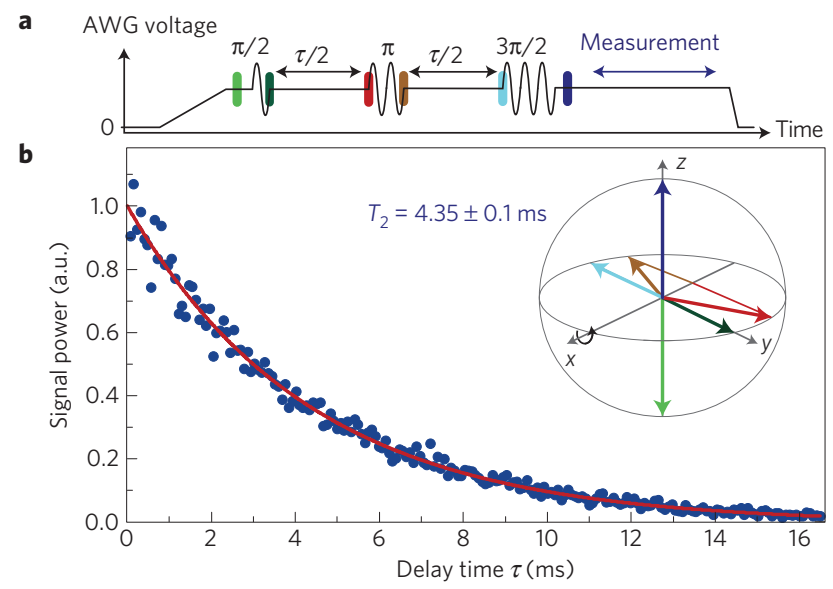

Figure $\mathbf{5}$ | Hahn echo. a, Pulse scheme. The system is adiabatically tuned from the initialization to the lower state. $A \pi / 2$-pulse creates a superposition state, and after a delay of $\tau / 2$ a $\pi$-pulse mirrors the state vector to the other half of the Bloch sphere. After another delay of $\tau / 2$, a $3 \pi / 2$-pulse is used to rotate to the upper state. $\mathbf{b}$, The inverse evolution of the system during the two delay times cancels out any broadening or slow precession effects, thus the system always ends up along the $z$ axis and no oscillation is observed (data shown in dark blue; fit in red). The Bloch sphere in the inset shows the state of the Bloch vector at selected times, which are marked in the same colour in $\mathbf{a}$.

axis. The resulting decay curve represents $T_{2}$, for which a value of $T_{2}=4.35 \pm 0.1 \mathrm{~ms}$ can be extracted from the fit in Fig. $5 \mathrm{~b}$.

The good agreement between $T_{2}$ and $T_{2}^{*}$ clearly shows that reversible elastic dephasing, for example caused by temporal and spatial enviromental fluctuations or spatial inhomogeneities, does not noticeably increase decoherence. The experiment is performed with the two hybrid modes occupied by billions of phonons, making it analogous to a many-spin NMR measurement, only that the macroscopic magnetization is replaced by the mode polarization and a single spin flip corresponds to the transfer of a single phonon between the two modes. But in contrast to the
NMR system, here all quasi-particles reside in the same collective mechanical mode and thus experience an identical environment (Supplementary Section SIII).

It is more surprising that the phase coherence time $T_{2}$ is equal to the average energy relaxation time $T_{1}$. This indicates the absence of measurable elastic phase relaxation processes in the nanomechanical system, such that the observed loss of coherence is essentially caused by the energy decay of the mechanical oscillation (Supplementary Section SIII). Earlier research ${ }^{20}$ suggests that the dominant relaxation mechanism in silicon nitride strings is mediated by localized defect states of the amorphous resonator material, described as two-level systems at low temperature. They facilitate energy relaxation by providing the momentum required to transform a resonator phonon into a bulk phonon. For this process to lead to elastic phase relaxation, an excited defect state would have to re-emit the phonon back into the resonator mode, which is extremely unlikely owing to the weak coupling between the two.

In conclusion, we demonstrate coherent electrical control of a strongly coupled $(\Omega \gg f / Q)$ classical nanomechanical two-level system, employing the pulse techniques well-known from coherent spin dynamics in the field of nanomechanics. Each superposition state of the two hybrid modes on the Bloch sphere can be addressed by a sequence of the described pulses. The presented system stands out by the finding that the elastic phase relaxation rate $\Gamma_{\varphi}$ is negligible compared with the energy decay rate $2 \pi f / Q$, leaving room for improvement of the coherence by means of increased quality factors.

The coherent manipulation schemes presented here allow the simulation of quantum systems using a classical two-level system ${ }^{2}$. Furthermore, in light of the recent breakthrough in ground-state cooling of nanomechanical resonators ${ }^{23-26}$, they can be directly transferred to quantum nanomechanical systems, where they open up new applications in quantum information processing. Not only can they be used as efficient interfaces for quantum state transfers in hybrid quantum systems ${ }^{27,28}$, but by creating coupled, quantized resonators ${ }^{29}$ quantum computations can be carried out directly using nanoelectromechanical two-level systems ${ }^{30}$.

Received 27 December 2012; accepted 8 May 2013; published online 2 July 2013

\section{References}

1. Nielsen, M. A. \& Chuang, I. L. Quantum Computation and Quantum Information (Cambridge Univ. Press, 2000).

2. Dragoman, D. \& Dragoman, M. (eds) Quantum-Classical Analogies (Springer, 2004).

3. Vandersypen, L. M. K. \& Chuang, I. L. NMR techniques for quantum control and computation. Rev. Mod. Phys. 76, 1037-1069 (2005).

4. Haroche, S. \& Raimond, J-M. Exploring the Quantum: Atoms, Cavities, and Photons (Oxford Univ. Press, 2006).

5. Hanson, R. \& Awschalom, D. D. Coherent manipulation of single spins in semiconductors. Nature 453, 1043-1049 (2008).

6. You, J. Q. \& Nori, F. Atomic physics and quantum optics using superconducting circuits. Nature 474, 589-597 (2011).

7. Bloch, F. Nuclear induction. Phys. Rev. 70, 460-474 (1946).

8. Yafet, Y. g factors and spin-lattice relaxation of conduction electrons. Solid State Phys. 14, 1-98 (1963).

9. Burkard, G., Koch, R. H. \& DiVincenzo, D. P. Multilevel quantum description of decoherence in superconducting qubits. Phys. Rev. B 69, 064503 (2004).

10. Faust, T. et al. Nonadiabatic dynamics of two strongly coupled nanomechanical resonator modes. Phys. Rev. Lett. 109, 037205 (2012).

11. Rabi, I. I. Space quantization in a gyrating magnetic field. Phys. Rev. 51, 652-654 (1937).

12. Ramsey, N. F. A molecular beam resonance method with separated oscillating fields. Phys. Rev. 78, 695-699 (1950).

13. Hahn, E. L. Spin echoes. Phys. Rev. 80, 580-594 (1950).

14. Spreeuw, R. J. C., van Druten, N. J., Beijersbergen, M. W., Eliel, E. R. \& Woerdman, J. P. Classical realization of a strongly driven two-level system. Phys. Rev. Lett. 65, 2642-2645 (1990).

15. Perisanu, S. et al. The mechanical resonances of electrostatically coupled nanocantilevers. Appl. Phys. Lett. 98, 063110 (2011). 
16. Okamoto, H., Kamada, T., Onomitsu, K., Mahboob, I. \& Yamaguchi, H. Optical tuning of coupled micromechanical resonators. Appl. Phys. Express 2, 062202 (2009).

17. Karabalin, R. B., Cross, M. C. \& Roukes, M. L. Nonlinear dynamics and chaos in two coupled nanomechanical resonators. Phys. Rev. B 79, 165309 (2009).

18. Kozinsky, I., Postma, H. W. C., Bargatin, I. \& Roukes, M. L. Tuning nonlinearity, dynamic range, and frequency of nanomechanical resonators. Appl. Phys. Lett. 88, 253101 (2006).

19. Okamoto, H. et al. Coherent phonon manipulation in coupled mechanical resonators. Nature Phys.http://dx.doi.org/10.1038/nphys2665 (2013).

20. Unterreithmeier, Q. P., Faust, T. \& Kotthaus, J. P. Damping of nanomechanical resonators. Phys. Rev. Lett. 105, 027205 (2010).

21. Faust, T., Krenn, P., Manus, S., Kotthaus, J. P. \& Weig, E. M. Microwave cavity-enhanced transduction for plug and play nanomechanics at room temperature. Nature Commun. 3, 728 (2012).

22. Rieger, J., Faust, T., Seitner, M. J., Kotthaus, J. P. \& Weig, E. M. Frequency and Q factor control of nanomechanical resonators. Appl. Phys. Lett. 101, 103110 (2012).

23. O'Connell, A. D. et al. Quantum ground state and single-phonon control of a mechanical resonator. Nature 464, 697-703 (2010).

24. Teufel, J. D. et al. Sideband cooling of micromechanical motion to the quantum ground state. Nature 475, 359-363 (2011).

25. Chan, J. et al. Laser cooling of a nanomechanical oscillator into its quantum ground state. Nature 478, 89-92 (2011).

26. Safavi-Naeini, A. H. et al. Observation of quantum motion of a nanomechanical resonator. Phys. Rev. Lett. 108, 033602 (2012)

27. Stannigel, et al. Optomechanical transducers for long-distance quantum communication. Phys. Rev. Lett. 105, 220501 (2010)
28. Meystre, P. A short walk through quantum optomechanics. Ann. Phys. 525, 215-233 (2013)

29. Brown, K. R. et al. Coupled quantized mechanical oscillators. Nature 471, 196-199 (2011)

30. Rips, S. \& Hartmann, M. J. Quantum information processing with nanomechanical qubits. Phys. Rev. Lett. 110, 120503 (2013).

\section{Acknowledgements}

Financial support by the Deutsche Forschungsgemeinschaft through Project No. Ko 416/18, the German Excellence Initiative through the Nanosystems Initiative Munich (NIM) and LMUexcellent, as well as the European Commission under the FET-Open project QNEMS (233992) is gratefully acknowledged. We thank G. Burkard for his comments on decoherence in a three-level system and H. Okamoto, I. Mahboob and

H. Yamaguchi for critically reading the manuscript.

\section{Author contributions}

J.R. and M.J.S. designed and fabricated the sample, T.F. conducted the measurements and analysed the data. T.F., J.P.K. and E.M.W. wrote the paper with input from the other authors. The results were discussed by all the authors.

\section{Additional information}

Supplementary information is available in the online version of the paper. Reprints and permissions information is available online at www.nature.com/reprints. Correspondence and requests for materials should be addressed to E.M.W.

\section{Competing financial interests}

The authors declare no competing financial interests. 